\title{
Additional diagnostic value of multiplane echocardiography over biplane imaging in assessment of mitral prosthetic valves
}

\author{
Francesco Faletra, Francesca De Chiara, Roberto Corno, Luca Passini
}

\begin{abstract}
Objective-To evaluate how often multiplane transoesophageal echocardiography yields new or complementary data in mitral prostheses in comparison with the exclusive use of biplane imaging.

Patients -73 consecutive patients with mitral prostheses who underwent multiplane transoesophageal echocardiograpy between January 1993 and December 1994.

Methods-Biplane images (transverse and longitudinal planes) and multiplane images (transverse, longitudinal, and intermediate planes) were recorded on two separate videotapes. The data provided by multiplane transoesophageal echocardiography were evaluated as (a) new data (abnormalities missed by biplane imaging); (b) complementary data (better delineating lesions already visualised by biplane imaging); or (c) redundant data (data already provided by biplane imaging).
\end{abstract}

Results-Multiplane transoesophageal echocardiography revealed new abnormalities in seven patients $(9 \cdot 5 \%)$ (thrombi in three and paraprosthetic leaks in the remaining four) and complementary data in nine $(12 \cdot 3 \%)$. In patients with paraprosthetic regurgitation, the possibility of continuously visualising the sewing ring by means of sequential angulations allowed the circumferential extension of the leak to be measured. In seven patients with paravalvar regurgitation who underwent surgery, the extension of the leak as measured by the multiplane approach closely corresponded with the surgical data.

Conclusions-In comparison with the exclusive use of biplane imaging, the multiplane approach added new or complementary data in a significant proportion of patients with mitral prostheses. The ability to obtain the sequential adjacent planes allowed a more reliable appraisal of the extension of the leak and other abnormalities.

(Heart 1996;75:609-613)

Keywords: transoesophageal echocardiography; biplane imaging; multiplane imaging; mitral prostheses

Transoesophageal echocardiography has become a well established diagnostic tool in the evaluation of mitral prostheses ${ }^{1}$; moreover, the introduction of the biplane mode has improved the definition of the anterior and posterior aspect of the sewing ring, thus increasing the diagnostic power of the technique. $^{2}$

The availability of a multiplane probe, which theoretically can yield a countless series of adjacent planes by rotating the transducer through a $180^{\circ}$ arc, has recently offered a great deal of flexibility in optimally aligning the scan beam to a specific anatomical structure. In this way, it is possible to choose those imaging planes that provide the maximum information. ${ }^{3-5}$ It has been suggested that multiplane transoesophageal echocardiography should further increase diagnostic accuracy ${ }^{6}$; however, its additional diagnostic value in the evaluation of mitral prostheses has not yet been systematically assessed in a large number of patients.

In this study, we analysed how often multiplane transoesophageal echocardiography (that is, all planes throughout an arc of $180^{\circ}$ ) yields additional useful clinical information in comparison with the exclusive use of the transverse $\left(0^{\circ}\right)$ and longitudinal $\left(90^{\circ}\right)$ planes of biplane imaging.

\section{Methods}

STUDY POPULATION

Between January 1993 and September 1994, 73 patients with mitral valve prostheses (51 women and 22 men; age 53, SD14, years; range 34-78) underwent multiplane transoesophageal examination. Sixty eight patients had mechanical mitral prostheses. Of these, 64 were tilting disc prostheses: 61 Sorin (Sorin Biomedica) and three Bjork-Shiley (Shiley Inc); and four were ball and cage prostheses (Starr-Edwards, American Edwards); five patients had biological mitral prostheses (Carpentier-Edwards, American Edwards). Twenty six patients had associated aortic prostheses and one an associated tricuspid and aortic prosthesis.

The transoesophageal study was performed for one or more of the following indications: (1) the need for the better delineation of prosthetic abnormalities diagnosed by transthoracic echocardiography, (2) mismatch between transthoracic echocardiographic and clinical data (that is, normal echocardiographic and Doppler data with worsening clinical status), (3) the presence of fever or haemolytic anaemia without any recognisable cause, (4) the identification of a cardiac source of emboli. 
New imaging data obtained using intermediate planes

\begin{tabular}{|c|c|c|c|c|}
\hline Patient No & Prosthesis type & Site & $\begin{array}{l}\text { Transverse and } \\
\text { longitudinal planes }\end{array}$ & Intermediate planes \\
\hline 1 & Biological & $\mathbf{M}$ & Normal & Thrombus in LAA $\left(51^{\circ}\right)$ \\
\hline 2 & Tilting disc & M/Ao & Normal & Posterior dehiscence \\
\hline 3 & Bileaflet & $\mathbf{M}$ & Abnormal regurgitation & Anterior dehiscence \\
\hline 4 & Biological & $\mathbf{M}$ & $\begin{array}{l}\text { Cusp thickening and } \\
\text { intraprosthetic regurgitation }\end{array}$ & $\begin{array}{l}\text { Cusp thickening and } \\
\text { intraprosthetic regurgitation. } \\
\text { Posterior dehiscence }\end{array}$ \\
\hline 5 & Bileaflet & $\mathbf{M}$ & Normal & Thrombus in LA $\left(118^{\circ}\right)$ \\
\hline 6 & Tilting disc & $\mathbf{M}$ & Anterior dehiscence & $\begin{array}{l}\text { Anterior dehiscence } \\
\text { thrombus on venticular } \\
\text { surface of the disc }\end{array}$ \\
\hline 7 & Tilting disc & M/Ao & Normal & Anterior dehiscence \\
\hline
\end{tabular}

M, mitral; M/Ao, mitral and aortic; LA, left atrium; LAA, left atrial appendage.

We also identified those patients in the study group who had undergone surgery for a dysfunctioning prosthesis and compared the surgical findings with the multiplane transoesophageal data.

TRANSOESOPHAGEAL EXAMINATION

We used a commercially available multiplane transoesophageal probe (Omniplane, HewlettPackard) composed of 64 elements, with a dual frequency ( 3.7 and $5 \mathrm{MHz}$ ) and pulsed, continuous wave, and colour Doppler capabilities. The probe was interfaced with the echocardiographic machine (HP Sonos 1500, Hewlett-Packard) and the operations at the console (on/off or pulsed, continuous, and colour Doppler modes, gain, depth, angle, zoom) were the same as those used with transthoracic transducers. The transducer encased within the tip of the shaft can be rotated through an arc of $180^{\circ}$ by pressing a button in the handle of the echoscope. The angle of the plane is indicated by a needle-like indicator located within an icon in the form of a semicircle on the screen.

The transoesophageal study was performed using a technique described in detail elsewhere. ${ }^{5}$ Briefly, all of the patients abstained

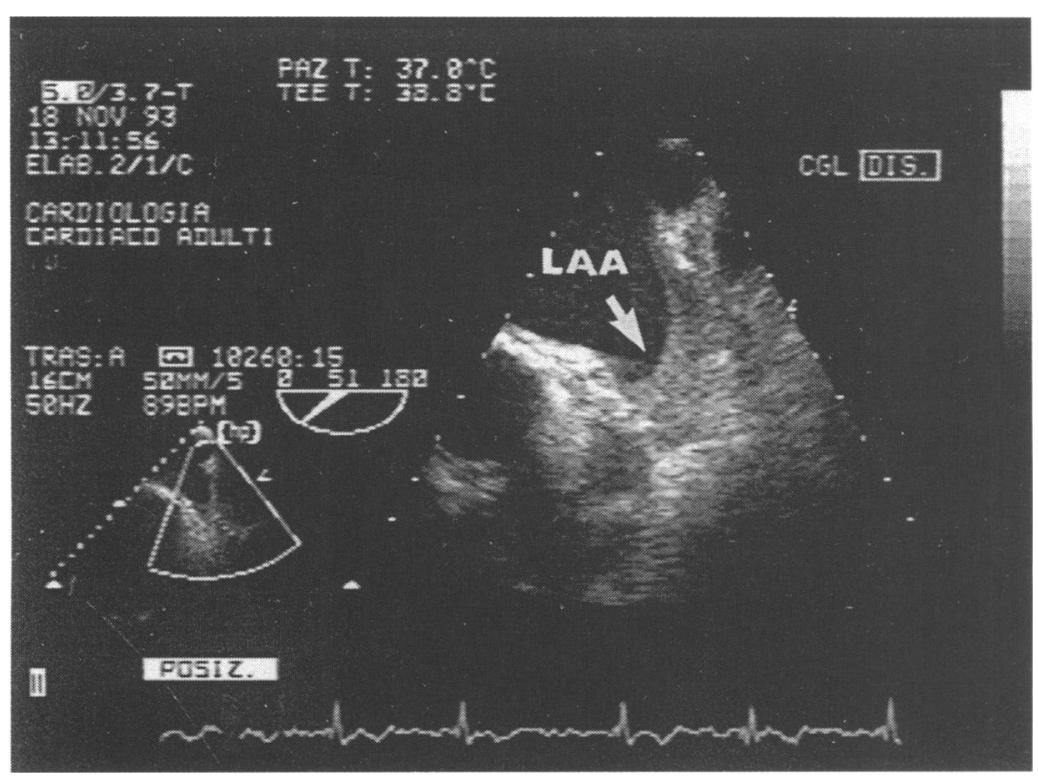

Figure $1 A$ thrombus in the left atrial appendage seen at $51^{\circ}$. The smooth surface of the thrombus and its concave configuration (arrow) probably prevented its detection with biplane imaging. $L A A$, left atrial appendage. from any oral intake for at least $4 \mathrm{~h}$ before the study and a history was taken to exclude the presence of possible oesophageal diseases. After the patient had given informed consent, the pharynx was sprayed with aerosolised lignocaine and intravenous midazolam (2.5-5 $\mathrm{mg}$ ) was given if necessary or requested. The probe was then inserted with the patient lying in the left lateral decubitus position.

The examination was started by scanning the prosthesis in the transverse $\left(0^{\circ}\right)$ plane at the level of the mid-oesophagus (approximately $25-30 \mathrm{~cm}$ from the incisors). At this depth a four-chamber and, by angling the transducer, a long axis view of the left ventricle were obtained. Subsequently, the transducer was rotated through $90^{\circ}$ in order to visualise the two-chamber view. Finally, by steering the ultrasound beam degree by degree a comprehensive scan of the whole circumference was obtained from the same position. Transgastric imaging was obtained following the same sequential steps (transverse $0^{\circ}$, longitudinal $90^{\circ}$, and intermediate planes) in all of the patients.

Adjustments made by advancing or withdrawing the transducer or by anteflexing, retroflexing, or rotating the probe were accomplished for optimising the imaging plane.

The biplane images and the full multiplane examination (that is, transverse, longitudinal, and intermediate planes) were recorded on two separate videotapes during the examination (in the first 15 studies the biplane images were rerecorded on a separate tape afterwards). Intermediate planes imaged during the rotation between the transverse and longitudinal plane were not recorded on the tape containing the biplane imaging part of the examination, with the exception of those planes ranging form $0^{\circ} \pm 10$ and $90^{\circ} \pm 10$, which were included in the biplane part of the analysis.

Two experienced physicians (neither of whom was involved in performing the examinations) separately analysed the two videotapes (being unaware of each other's evaluation). Once prosthetic anatomy and function were established with both approaches, the data obtained from the full multiplane examination were compared with those of the biplane images. The observers were then asked to judge whether full multiplane imaging provided (a) new data (that is, abnormalities missed by biplane imaging), (b) complementary data (that is, lesions already visualised by biplane imaging but better delineated by intermediate planes), or (c) redundant data (data already provided by biplane imaging).

In the case of any disagreement concerning this judgement, a consensus was reached with a third physician.

\section{Results}

Complete multiplane transoesophageal echocardiography was performed in all patients; none of the patients had major difficulty in swallowing the probe. The duration of exami- 


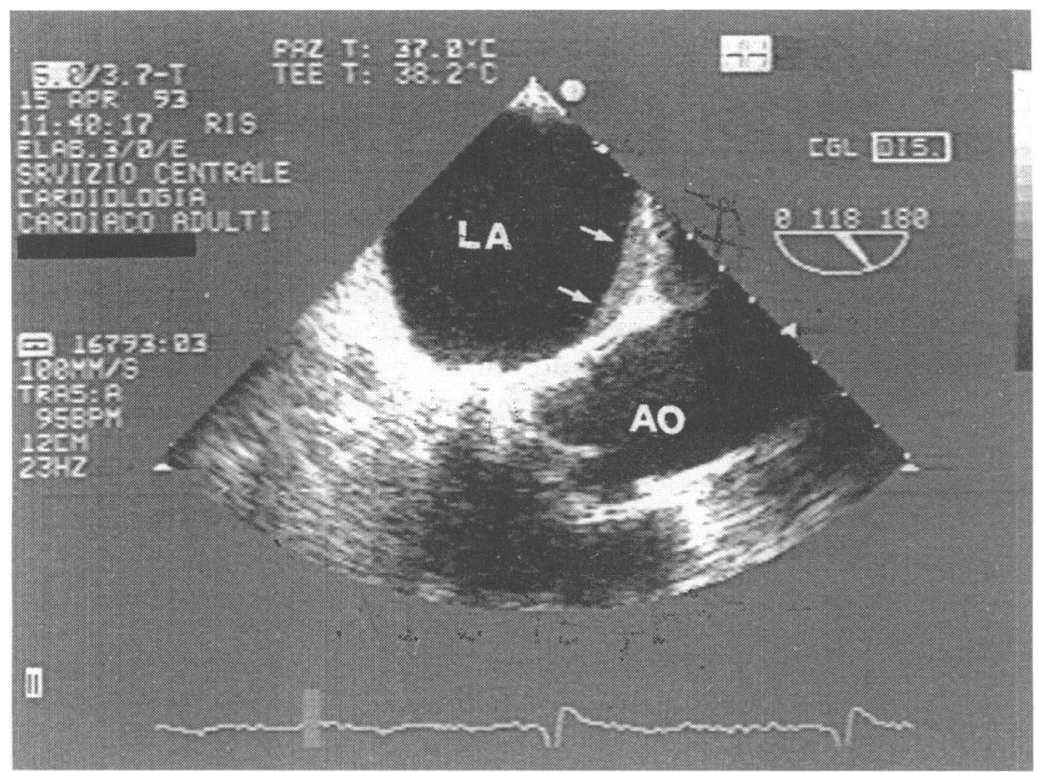

Figure $2 A$ thrombus layered on the left atrial wall, imaged at $118^{\circ}$ (arrows). Its smooth surface and thinness probably prevented its detection with biplane imaging. $L A$, left atrium; Ao, aorta.

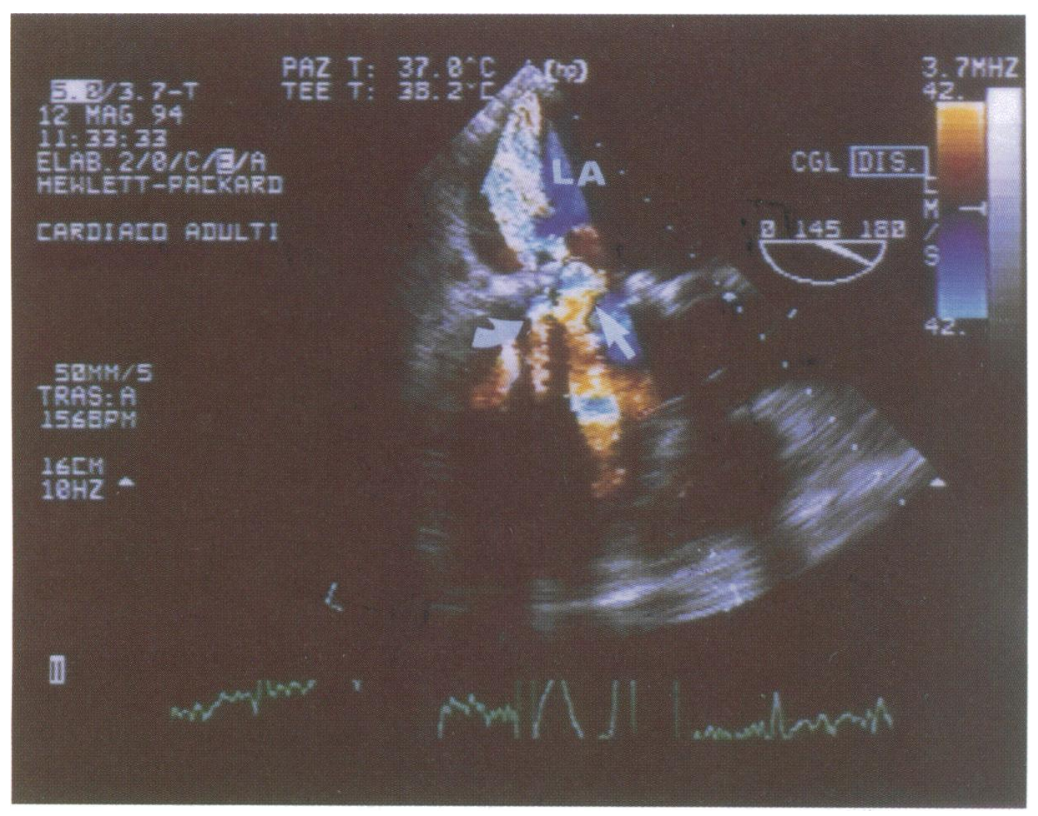

Figure 3 Patient with severe intraprosthetic regurgitation due to cusp degeneration in whom the intermediate planes revealed an additional paraprosthetic jet which merged with the intraprosthetic jet immediately after their origin. The two separate flow convergence regions (arrows) seen using intermediate planes helped in detecting the two jets. In this patient the results of multiplane transoesophageal echocardiography were confirmed at surgery. $L A$, left atrium. biplane imaging was discovered between $47^{\circ}$ and $68^{\circ}$ and between $103^{\circ}$ to $107^{\circ}$. In one patient with a biological prosthesis, a thickening and degeneration of the cusps with severe intraprosthetic regurgitation was seen at biplane imaging; however, by scanning from 106 to 153 degrees, a large paraprosthetic jet was discovered, which was seen to merge with the intraprosthetic jet immediately after its origin (fig 3). Finally, biplane imaging in one patient showed a small turbulent jet in the left atrium; however, the paraprosthetic origin of the jet was only identified by the intermediate planes ranging from $58^{\circ}$ to $63^{\circ}$.

In nine patients $(12.3 \%)$, the intermediate planes added complementary data. In one patient, a thrombus was strongly suspected at biplane imaging but, at $59^{\circ}$, it was seen to be partially occluding the orifice of the prosthesis and preventing the proper opening and closing of the disc (fig $4 \mathrm{~A}, \mathrm{~B}$ ). In two patients with paraprosthetic leaks, the origin of the jet was better defined by the intermediate planes as coming from outside the sewing ring. In the remaining six patients with a paraprosthetic leak diagnosed by biplane imaging, the ability to scan through an arc of $180^{\circ}$ allowed the extension of the leak to be measured (ranging from $18^{\circ}$ to $65^{\circ}$ degrees) (fig $5 \mathrm{~A}, \mathrm{~B}$ ).

In the remaining 57 patients $(78 \cdot 1 \%)$, the intermediate plane did not add any new or complementary information to that provided by biplane imaging.

Finally, the presence of an additional prosthesis in the aortic position did not significantly interfere with the reader's judgement of mitral prosthesis function in either the biplane or the multiplane part of the study.

In the nine patients in whom surgical data were available, a complete correspondence between the surgical and multiplane echocardiographic findings was found: surgery confirmed a thrombus preventing proper opening and closing of the disc in two patients, a flail leaflet and a paravalvar leak in a patient with a bioprosthesis, and a paravalvar leak in the remaining six patients. In all of the seven patients with paraprosthetic regurgitation, the site and circumferential extension of the leak provided by scanning the circumference of the sewing ring closely corresponded with the surgical data.

\section{Discussion}

Since it overcomes the limitations of conventional transthoracic echocardiography that result from the reverberations and flow masking of non-biological materials, transoesophageal echocardiography has become an essential part of the comprehensive cross sectional/Doppler echocardiographic examination in patients with a suspected dysfunction of mitral prostheses. ${ }^{1}$ However, single plane transoesophageal echocardiography has a restricted number of sections available, and a complete circumannular inspection of the prosthesis is often not possible. Moreover, it requires considerable manipulation of the transducer to obtain appropriate planes and 
the desired plane often cannot be imaged. The advent of biplane transducer capability was an important development in the assessment of mitral prostheses, as it yielded additional lon-
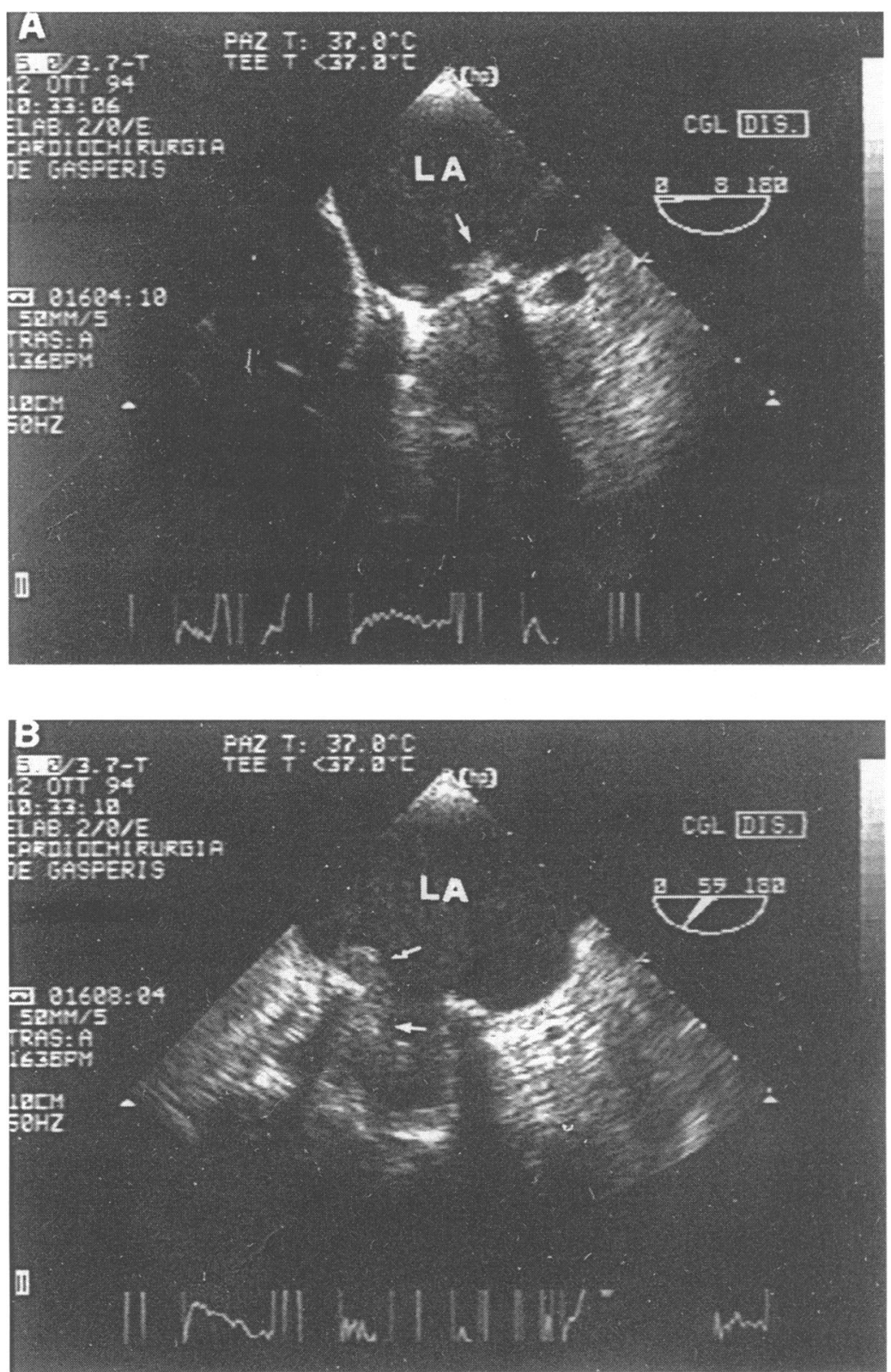

Figure 4 Patient with an obstructed prosthesis. A thrombus was strongly suspected at biplane imaging $(A)$ but at $59^{\circ}$ it was seen to be partially occluding the orifice of the prosthesis and preventing the proper opening and closing of the disc $(B)$. The obstruction (and pathological intraprosthetic regurgitation) due to the presence of thrombus was confirmed at surgery. gitudinal planes which allowed for the easier identification of both anterior and posterior lesions of the sewing ring. ${ }^{2}$

It is clear that the wide selection of planes provided by multiplane transoesophageal echocardiography allows the easier acquisition of the most appropriate echocardiographic images. Previous studies have addressed this question, in a general population ${ }^{67}$ or in mixed aortic and mitral prostheses ${ }^{8}$ : Tardiff et $a l^{6}$ studied 80 patients with a variety of cardiac disorders and found that multiplane echocardiography identified more lesions in $10 \%$ of patients and delineated abnormalities better in $39 \%$. However, they referred to only one patient with a mitral prosthesis, in whom the intermediate planes provided better delineation of a layered thrombus in the left atrium. A similar population of 103 patients was studied by Flachskampf $e t \mathrm{al}^{7}$ : a clear separation of paravalvar leakage from transvalvar regurgitation was found in nine patients using biplane imaging and in 14 patients using intermediate planes, with a $36 \%$ increase in imaging information. In a more recent study ${ }^{8}$ in which 35 mitral and 33 aortic prostheses were examined, the same investigators found the type of regurgitation not clearly defined by biplane imaging in 19 patients; in only three cases (all in the aortic position) the type of regurgitation remained unclear after multiplane examination.

The present study, which included the largest number of patients with mitral prostheses, is consistent with the above mentioned papers and showed that, in comparison with the exclusive use of biplane imaging, the full multiplane approach provided new or complementary data in a significant proportion of patients (16 of 73 patients; $22 \%$ ). In seven of these, the intermediate planes revealed things missed by biplane imaging. In one of the three patients with thrombi, the thrombus was located in the left atrial appendage and its particular configuration (it had a smooth and concave surface similar to the shape of the atrial appendage) probably prevented its detection by biplane imaging, as was probably also the case in the second patient, in whom the thrombus was very thin and layered on the left atrial wall (figs 1 and 2). In the remaining patient, the thrombus was located on the ventricular surface of the disc and reverberations
Figure 5 Patient with paraprosthetic regurgitation. The jet is imaged passing through the sewing ring, starting at $53^{\circ}$ (A) and finishing at $71^{\circ}$ (B). The extension of the leak was therefore $18^{\circ}$. The site and extension of the leak had a close correspondence with those discovered at surgery.
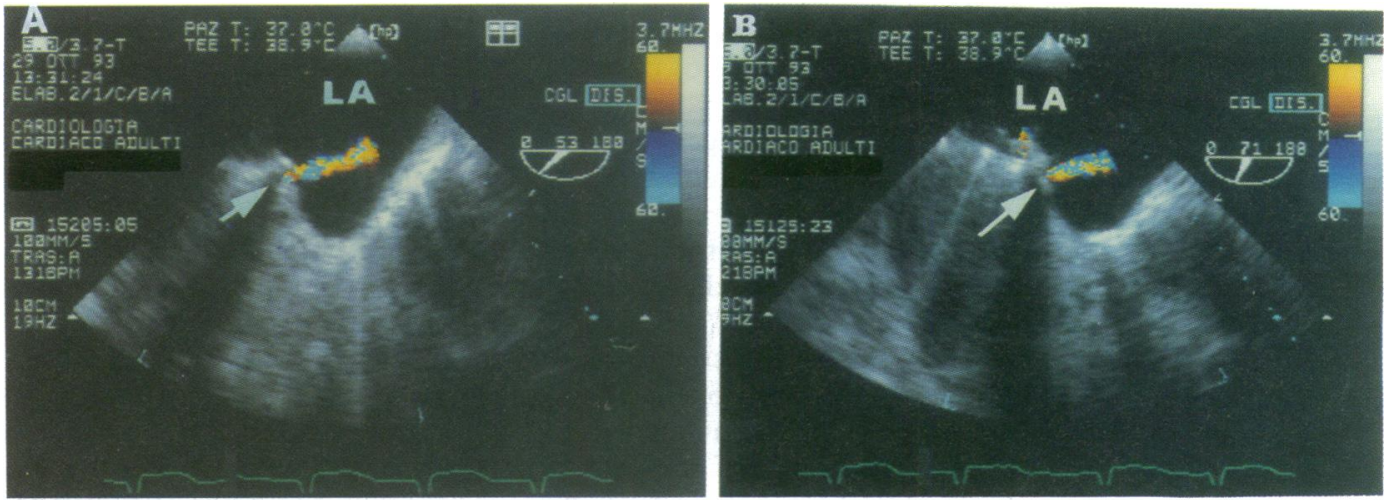
prevented its identification by biplane imaging. In three of four patients with paraprosthetic regurgitation, the jet was thin and its paraprosthetic origin was missed by biplane imaging just because it was out of the biplane scan planes. In the fourth patient it was the merging of the two jets immediately after their origin that probably prevented their distinction by biplane imaging; by using intermediate planes, two separate flow convergence regions on the ventricular surface of the sewing ring were clearly imaged (fig 3 ).

In the remaining nine patients, multiplane transoesophageal echocardiography added complementary data. Of particular interest to us was its ability to establish the extension of the leak over the sewing ring: once the best and most stable plane for imaging the paraprosthetic jet was ascertained, the rotation of the scan plane degree by degree enabled us to detect both the initial and the final point where the jet is imaged passing through the ring and, therefore, to measure its circumannular extension (fig 5). We also found a close correspondence between this measurement and the estimate of the extension of the leak at surgery; this information could offer an alternative way of assessing the severity of the leak, regardless of the extension of the regurgitant jet.

In none of the seven patients in whom intermediate planes provided new data did this have a critical impact on the management. In three of the four patients in whom multiplane transoesophageal echocardiography revealed a paravalvar leak missed by biplane imaging, the regurgitation was trivial and clinically silent. The patients did not require reoperation and were scheduled for clinical and echocardiographic follow up. The fourth patient had an associated severe intraprosthetic regurgitant jet due to the degeneration of a biological prosthesis, and therefore would have undergone surgery in any case. In a fifth patient, in whom an obstruction associated with a paraprosthetic leak was diagnosed by biplane imaging, the detection of a thrombus on the ventricular surface of the disc by means of intermediate planes provided additional information, but did not change the surgical picture. Finally, the presence of thrombi in the left atrial appendage and in the left atrium, missed by biplane imaging in the last two patients, did not change the therapeutic approach in so far as both patients were already under correct anticoagulant treatment.
LIMITATIONS OF THE STUDY

The ideal way to compare biplane versus multiplane imaging would be to perform a double intubation in every patient. However, since patients cannot be examined sequentially with two different probes, we circumvented this limitation by recording the biplane images and the full multiplane examination on two different videotapes. In this way we were able to read the two tapes as if they came from two different studies.

Although care in obtaining intermediate planes by forcing the tip of the probe either laterally or anteriorly and posteriorly was taken during the biplane part of the examination (as if a biplane transducer was being used), we are aware that the lack of a proper or more extensive manipulation of the probe cannot be excluded. To compensate for the information potentially missed, we applied the same expedient used by Tardiff et al, ${ }^{6}$ including $\pm 10^{\circ}$ degree additional planes in the biplane recorded part.

\section{CONCLUSION}

In comparison with biplane imaging, the multiplane approach adds new or complementary data in a significant percent of patients with mitral prostheses. The ability to obtain sequential adjacent planes allowed a more reliable appraisal of the extension of the leak and other abnormalities.

1 Khanderia BK, Seward JB, Oh JK, et al. Value and limitations of transesophageal echocardiography in assessment of mitral valve prostheses. Circulation 1991;68:689-90.

2 Groundstroem K, Rittoo D, Hoffman P, Bloomfield P, Sutherland GR. Additional value of biplane transoesophageal imaging in assessment of mitral valve prostheses. Br Heart f 1993;70:259-65.

3 Pandian NG, Hsu TL, Schwartz SL, et al. Multiplane transesophageal echocardiography. Imaging planes, echocardiographic anatomy and clinical experience with a prototype phased array omniplane probe. Echocardiography 1992;9:649-66.

4 Roelandt J, Thomson I, Vletter W, et al. Multiplane transesophageal echocardiography: latest evolution in a imaging revolution. $\Im$ Am Soc Echocardiography 1992;5:361-7.

5 Seward JB, Khandheira BK, Freeman WK, et al. Multiplane transesophageal echocardiography: image orientation, examination technique, anatomic correlation and clinical applications. Mayo Clin Proc 1993;68: 523-51.

6 Tardiff JC, Schwartz SL, Vannan MA, Cao QL, Pandian NG. Clinical usefulness of multiplane transesophageal echocardiography: comparison to biplane imaging. $\mathrm{Am}$ Heart $\mathcal{f}$ 1994;128:156-66

7 Flachskamp FA, Hoffmann R, Verlande M, Schneider W, Ameling W, Hanrath P. Initial experience with a multiplane transesophageal echotransducer: assessment of plane transesophageal echotransducer: assessm
diagnostic potential. Eur Heart $₹$ 1992;13:1201-6.

8 Flachskamp FA, Hoffmann R, Frank EA, Job FP, Schondube FA, Messmer BJ, et al. Does multiplane Schondube FA, Messmer BJ, et al. Does multiplane transesophageal echocardiography improve the assessment of prosthetic valve regurgitation? $\mathcal{f} A m$ Soc 